\title{
Análisis de diversidad genética en tres poblaciones de llamas (Lama glama) del noroeste argentino
}

\author{
Analisis of genetic diversity in three llama (Lama glama) populations \\ from north-western Argentina
}

\begin{abstract}
ANA V. BUSTAMANTE ${ }^{1}$, MARÍA L. MATÉ ${ }^{1}$, HUGO E. LAMAS ${ }^{2}$, GUILLERMO GIOVAMBATTISTA ${ }^{3}$, ANDRÉS ZAMBELLI ${ }^{1}$ \& LIDIA VIDAL-RIOJA ${ }^{1 *}$
\end{abstract}

\footnotetext{
${ }^{1}$ Instituto Multidisciplinario de Biología Celular (IMBICE), Casilla de Correos 403, Código Postal 1900, La Plata, Buenos Aires, Argentina

${ }^{2}$ Instituto de Biología de la Altura (INBIAL)

${ }^{3}$ Centro de Investigaciones en Genética Básica y Aplicada (CIGEBA);

* e-mail para correspondencia: lvidalrioja@imbice.org.ar
}

\begin{abstract}
RESUMEN
Este trabajo describe la variabilidad genética actual de tres poblaciones de llamas (Lama glama) del noroeste argentino (NOA), afectadas a la producción de fibra. Originariamente, las tropas fueron una única población la cual fue subdividida hace 10 años. Se estudiaron muestras de ADN de 77 animales mediante amplificación por PCR de 12 loci microsatélite con cebadores específicos de llama. La alta variabilidad genética comprobada se sustenta en el hallazgo total de 140 alelos diferentes, 9 a 16 alelos por locus y rangos de heterocigosidad observada y esperada por locus de 1 a 0 y 0,9 a 0,47 , respectivamente. Diecinueve de treinta y seis pruebas de equilibrio de Hardy-Weinberg mostraron desvíos significativos $(P<0,05)$ debidos a una deficiencia de heterocigotos que se correspondería con el comportamiento poligínico natural de la especie. El nivel de diferenciación genética observado entre los tres planteles, moderado (Fst $=0,076 ; \mathrm{P}=0,000$ ) pero significativo de subestructura, podría atribuirse a la introducción de progenitores machos de otras regiones geográficas, en el momento de subdividir la población original. La presencia de 44 alelos privados, distribuidos entre las tres tropas permitiría diseñar apareamientos dirigidos a mejorar la diversidad genética mediante el intercambio de nuevas variantes alélicas. La transferencia a los productores, de los resultados de este trabajo, beneficiará la adopción de futuras estrategias de manejo adaptadas a la situación particular de cada plantel.
\end{abstract}

Palabras clave: camélidos sudamericanos, diversidad genética, estructura poblacional, marcadores microsatélite, manejo.

\begin{abstract}
The current genetic variability of three llama (Lama glama) management units from the northwestern Argentine (NOA) was analyzed. The troops, originally comprised a unique population that 10 years ago was divided into the current three. The DNA of 77 animals was studied by PCR amplification of 12 loci using microsatellite primers specific of Lama glama. A high level of genetic variability is sustained by the finding of one hundred and forty total alleles, a range of 9 to 16 allele number per locus and observed and expected hetrozygosities per locus varying from 1 to 0 and 0.9 to 0.47 , respectively. Distributed within the three troops 44 private alleles were detected and proposed for uses such as to exchange new allelic variants. Hardy Weinberg Equilibrium test for each locus within each population showed significant deviation $(\mathrm{P}<0.05)$ due to heterozygotes deficiency which may obey to the natural polygynic behaviour of the species. A moderated genetic differentiation between populations (Fst $=0.076 ; \mathrm{P}=0.000$ ) may be explained by the introduction of foreing males parents at the moment of the original population subdivision. Transference to breeders of the data here obtained may be important in future management programmes.
\end{abstract}

Key words: Lama glama, population history, genetic diversity, population structure, microsatellite markers, management. 


\section{INTRODUCCIÓN}

La llama (Lama glama) es el camélido sudamericano doméstico más grande y mejor adaptado a un amplio rango de condiciones medioambientales. Luego de su domesticación, a partir del guanaco silvestre (Lama guanicoe) en la puna peruana, hace aproximadamente 6.000 años (Wheeler 1983) fue llevada por el habitante nativo a otras regiones sudamericanas, extendiendo así su área de distribución. A partir de 1532, con la colonización española y la introducción de ganados foráneos, los rebaños nativos fueron diezmados y desplazados a la puna de gran altura (3.800-5.000 $\mathrm{m}$ de altitud), donde los animales europeos no prosperaban (Novoa 1984). Por otra parte, los eventos de introgresión en los camélidos sudamericanos probablemente hayan sido recurrentes, con una mayor frecuencia durante y luego de la colonización española. Si a este fenómeno se le suma la drástica reducción de las poblaciones de camélidos domésticos asociada a la conquista y la consecuente merma en la calidad de la fibra, no sería erróneo presumir un empobrecimiento genético de la especie (Kadwell et al. 2001). A diferencia de otras especies domésticas, no existen registros escritos asociados a la crianza de estos animales, debido en gran medida, a la desaparición, durante la colonización, del conocimiento tradicional oral de los pastores (Wheeler 1995). Se estima que la población actual mundial de llamas alcanza las 3.3 millones de cabezas de las cuales Argentina alberga un 4,05\% siendo el noroeste argentino (NOA) la zona de mayor concentración de llamas del país (www.portalagrario.gov.pe). La cría de estos animales está dirigida a la producción de fibra y las técnicas de manejo de los rebaños aunque conservan parte de la tradición cultural de la zona tienen un alto componente de técnicas europeas. La selección dentro de los rebaños se realiza en base a rasgos fenotípicos y, si bien los pastores tradicionalmente admiten la existencia de híbridos entre llamas y alpacas, no siempre es posible reconocer estos animales basándose solo en su fenotipo (Kadwell et al. 2001).

En los últimos años la conservación de razas y/o poblaciones de distintas especies ha cobrado gran auge porque, entre otros factores, la pérdida de diversidad genética disminuye la capacidad de recuperar especies amenazadas y mantener y mejorar el rendimiento de otras incluidas en el circuito productivo. Para ello es necesario conocer la variabilidad genética y su distribución en las poblaciones, así como identificar alelos raros que indiquen la presencia de variantes genéticas únicas (González-Candelas et al. 2000, Aranguren-Méndez et al. 2001). Con excepción de caracteres como color de capa y calidad de fibra, poco se conoce de la variación genotípica de las llamas actuales siendo esta información básica para la evaluación y conservación de estos recursos genéticos. Frente a la reciente exportación de alpacas y llamas andinas, urge la necesidad de llevar a cabo un estudio orientado a la documentación de la variabilidad genética de estas especies a fin de diseñar planes de manejo tendientes a conservar este importante reservorio genético. A partir de estudios previos de caracterización de especies de camélidos sudamericanos (Wheeler et al. 2001, Sarno et al. 2001, Bustamante et al. 2002, Sarno et al. 2004, Maté et al. 2005) es posible inferir la existencia de una marcada diferencia en los niveles de diversidad genética y estructura poblacional entre las especies silvestres y domésticas de camélidos sudamericanos, las que serían consecuencia de la historia, dinámica y manejo poblacional. Los datos sobre el conocimiento histórico de las poblaciones analizadas es de gran importancia para la interpretación de factores que puedan afectar la diversidad y estructura genética de las poblaciones.

Los marcadores de ADN microsatélite son herramientas de uso corriente para evaluar la variabilidad y estructura genética de las poblaciones. En este trabajo se tipificaron por PCR 12 loci de ADN microsatélite en tres planteles de llamas manejadas con técnicas tradicionales y de las cuales se cuenta con información histórica. El objeto del trabajo fue dar respuesta a los siguientes interrogantes: (i) ¿cuánta variabilidad genética presentan las poblaciones de llamas del NOA estudiadas?, (ii) ¿qué factores pueden haber afectado la variabilidad genética?, y (iii) ¿qué tipo de manejo debería implementarse para mantener y/ o aumentar la variabilidad? 


\section{MATERIALES Y MÉTODOS}

\section{Planteles estudiados}

Los animales estudiados pertenecen a los planteles históricamente relacionados de Colonia Santa Isabel, Establecimiento Los Pioneros y Campo de Llampa Jaran, cuyos respectivos grupos fundadores procedieron de la subdivisión de una única población ubicada en la Reserva Laguna de Pozuelos de la Comisión Municipal de Cieneguillas en la provincia de Jujuy de Argentina. Desde principios del siglo $\mathrm{XX}$ esta población, perteneciente a un establecimiento familiar, fue criada en forma extensiva y seleccionada para la producción de fibra. A principios de la década del 90, la sucesión familiar de los campos llevó a la subdivisión de la población original en tres tropas, las cuales fueron enriquecidas mediante la introducción de machos reproductores de diferentes regiones geográficas del país y países vecinos. En la actualidad llevan 10 años de reproducción aislada sin la acción de machos ajenos a la tropa (Fig. 1).

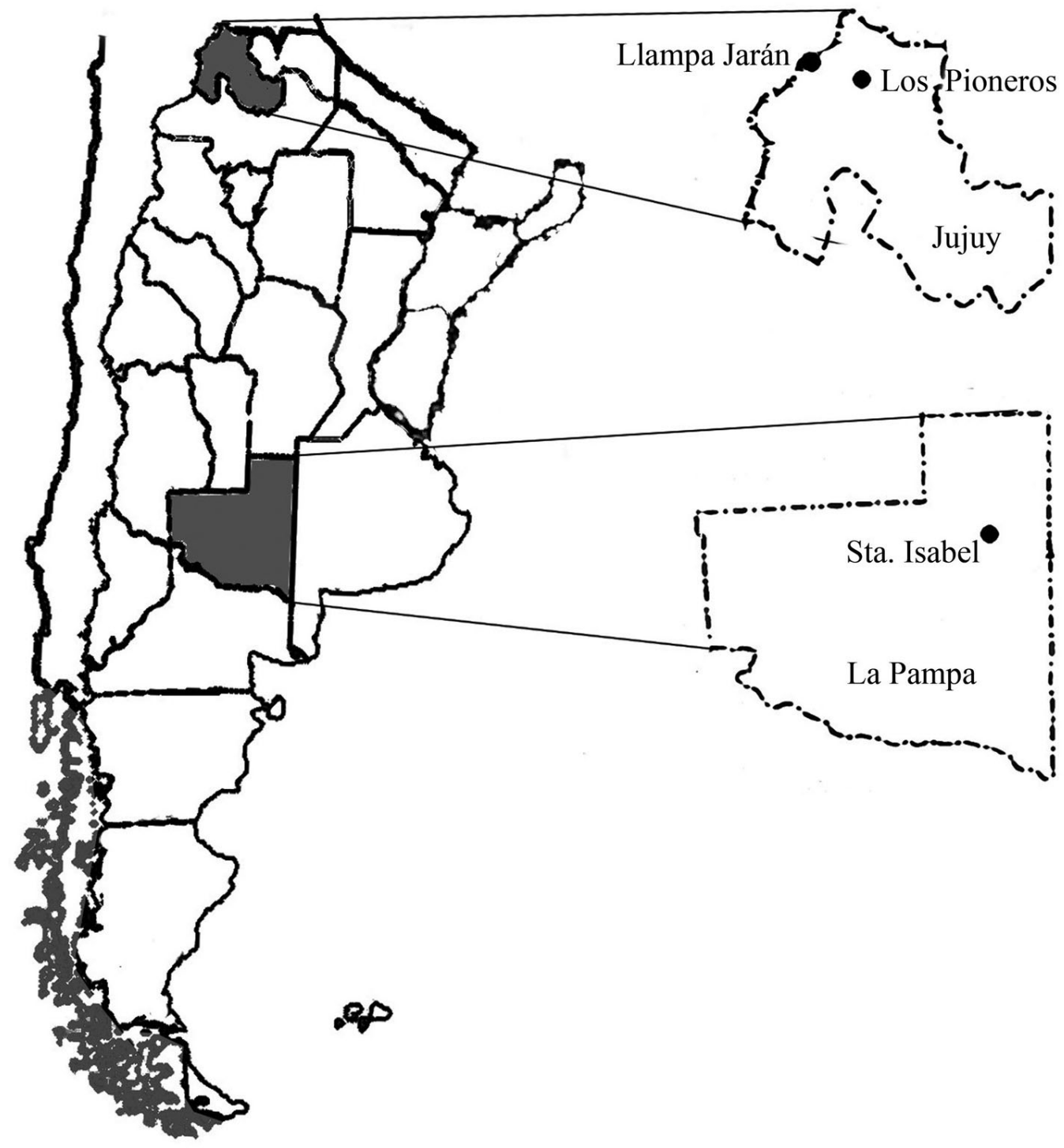

Fig. 1: Ubicación geográfica de los sitios de muestreo.

Geographic localization of the sampled places. 


\section{Establecimiento Los Pioneros (LP)}

Localizado en la Reserva Laguna de Pozuelos, ubicada a $3.600 \mathrm{~m}$ de altitud y $35 \mathrm{~km}$ al oeste de La Quiaca, provincia de Jujuy, Argentina (Fig. 1). En su origen la tropa fue enriquecida con la introducción de machos reproductores de Bolivia. Los animales son criados en forma extensiva y los cruzamientos están dirigidos a la producción de fibra por lo que el plantel sufre una fuerte presión de selección artificial. El aspecto fenotípico de los planteles evidencia la selección dirigida hacia animales de color blanco y tipo Suri.

\section{Campo de Llampa Jaran (LlJ)}

Ubicado a $4.600 \mathrm{~m}$ de altitud, a $33 \mathrm{~km}$ de la Comisión Municipal de Cusi Cusi, a $150 \mathrm{~km}$ al suroeste de La Quiaca y $150 \mathrm{~km}$ al noroeste de Abra-Pampa, provincia de Jujuy, Argentina. La distancia geográfica entre este plantel y el de Los Pioneros es de $115 \mathrm{~km}$ aproximadamente y se encuentra aislada geográficamente por la Sierra de Santa Catalina y el Río Grande de San Juan. En su origen, la población de este plantel fue incrementada mediante la introducción de animales de diferentes zonas de la provincia de Jujuy. Actualmente, es criado en forma extensiva y el aspecto fenotípico es mucho más rústico que el de Los Pioneros; las tropas están compuestas por animales de colores variados y con un alto porcentaje del tipo Huacaya.

\section{Colonia Santa Isabel (SI)}

La tropa de llamas de esta colonia actualmente pertenece a la Secretaría de Recursos Naturales de la Provincia de La Pampa y se cría en semicautividad en la ciudad de Santa Rosa, Provincia de La Pampa, Argentina. El núcleo fundador fue extraído en el año 1993 del establecimiento de cría Los Pioneros descrito arriba.

\section{Obtención de ADN}

El ADN genómico se extrajo a partir de 77 muestras de sangre fresca tomadas al azar de: Establecimiento Los Pioneros, $\mathrm{n}=30$; Campo de Llampa Jaran, $\mathrm{n}=22$; Colonia Santa Isabel, $\mathrm{n}=25$, siguiendo las técnicas descriptas en Bustamante et al. (2002).

\section{Loci microsatélite analizados}

Mediante la técnica de PCR se amplificaron 12 loci con los siguientes marcadores de ADN microsatélite específicos de llama LAB1, LAB3, LAB6, LAB7, LAB13 (Bustamante et al. 2003); LAB14, LAB15, LAB16, LAB17 (número de acceso al GenBank AY661538AY661541) y LCA56, LCA65 y LCA77 (Penedo et al. 1999). La amplificación se realizó en mezclas de reacción de $15 \mu 1$ conteniendo $50 \mathrm{ng}$ de ADN, 1,5-2,5 $\mathrm{mM}$ de $\mathrm{MgCl}_{2}, 10 \mathrm{mM}$ de Tris, $50 \mathrm{mM}$ de $\mathrm{KCl}, 200$ $\mu \mathrm{M}$ de cada dNTP, 12,5- 6,25 pmol de cada primer y 0,5 unidades de Taq ADN polimerasa (Promega, Madison, Wisconsin, USA). Se realizaron 35-40 ciclos, dependiendo de cada locus, con desnaturalización a $94{ }^{\circ} \mathrm{C}$ por $1 \mathrm{~min}$, hibridación a $48-56{ }^{\circ} \mathrm{C}$ por 1 min y elongación a $72{ }^{\circ} \mathrm{C}$ por $1 \mathrm{~min}$. Los productos de PCR se separaron en geles de poliacrilamida desnaturalizante al $6 \%(19: 1)$ 1x TBE. Como referencia de tamaño molecular se utilizaron escaleras de alelos conocidos construidas en el laboratorio.

\section{Análisis estadístico}

La diversidad genética se analizó, desde el punto de vista de la variedad alélica, mediante el cálculo del número medio de alelos por locus (NMA), los alelos privados por población (APP), el número de alelos detectados por población (A), el número total de alelos detectados $\left(\mathrm{A}_{\text {total }}\right)$ y a nivel de la proporción de los diferentes genotipos dentro de las poblaciones, mediante la diversidad genética $\left(h_{e}\right)$ y heterocigosidad observadas $\left(h_{o}\right)$ por locus en cada población y diversidad genética $\left(\mathrm{H}_{\mathrm{e}}\right)$ y heterocigosidad observada $\left(\mathrm{H}_{\mathrm{o}}\right)$ medias por población utilizando los algoritmos incluidos en el programa Exel Microsatellite Tools (Park 2001). Las desviaciones del equilibrio de Hardy-Weinberg (H-W) se evaluaron mediante el método exacto implementado en el programa GENEPOP versión 3.2 (Raymond \& Rousset 2000). Los niveles de significancia corregidos para el desvío de $\mathrm{H}-\mathrm{W}$ en cada población, se basaron en la técnica secuencial de Bonferroni para pruebas simultáneas (Rice 1995). La probabilidad de existencia de alelos nulos se estimó mediante el programa CERVUS 2.0 
(Marshall et al. 1998). La estructura y diferenciación genética entre las poblaciones se calculó mediante los parámetros $\mathrm{F}$ y Rho El índice Rho se basa en el modelo de mutaciones por pasos o Step Wise Model (SMM) y tiene en cuenta, además de las frecuencias alélicas, el tamaño de los alelos. Ambos cálculos se realizaron en forma global por marcador, para todas las poblaciones y todos los marcadores (GENEPOP versión 3.2a, Raymond \& Rousset 2000). También se realizó un Análisis Molecular de la Varianza (AMOVA) entre y dentro de los planteles utilizando dos métodos diferentes. Por un lado, el AMOVA se analizó a través de la variable "número de alelos diferentes $\left(\mathrm{F}_{\mathrm{ST}}\right)$ " y por otra parte a través de la "suma cuadrática de la diferencia entre los tamaños de los alelos $\left(\mathrm{R}_{\mathrm{ST}}\right)$ ". (ARLEQUIN versión 2.0, Excoffier 1992).

\section{RESULTADOS}

La diversidad genética medida en términos de variabilidad alélica, $\mathrm{H}_{\mathrm{e}}, \mathrm{H}_{\mathrm{o}}$ y $\mathrm{h}_{\mathrm{e}}, \mathrm{h}_{\mathrm{o}}$, mostró todos los loci analizados altamente polimórficos. En la muestra estudiada se observaron $140 \mathrm{~A}_{\text {total }}$ en los 12 loci tipificados, variando el A entre 92 y 100 . El $A_{\text {total }}$ por locus varió entre nueve (LAB6, LAB15, LCA65) y 16 (LAB3), mientras que dentro de cada población, el número de alelos por locus varió desde dos para LAB6 a 14 para LAB1 en la población de Llampa Jaran (Tabla 1). La $\mathrm{h}_{\mathrm{o}} \mathrm{y} \mathrm{h}_{\mathrm{e}}$ por locus varió de uno a cero y de 0,9 a 0,47 , respectivamente. $\mathrm{La} \mathrm{H}_{\mathrm{e}}$ por población fue similar en las tres poblaciones (Tabla 2). En total se observaron 44 alelos privados, de los cuales 18 corresponden a la población de LLJ, 15 a la población de SI y 11 a LP. Cabe

TABLA 1

Estadísticos de diversidad genética calculados por locus por población

Statistics of genetic diversity by locus by population

\begin{tabular}{|c|c|c|c|c|c|c|c|c|c|c|c|c|c|}
\hline \multirow[t]{2}{*}{ Población } & \multicolumn{12}{|c|}{ Locus } & \multirow[t]{2}{*}{ Total } \\
\hline & LAB 1 & LAB 3 & LAB 6 & LAB 7 & LAB 13 & LAB 14 & LAB 15 & LAB16 & LAB 17 & LCA56 & LCA65 & LCA77 & \\
\hline \multicolumn{14}{|l|}{ SI } \\
\hline $\mathrm{n}$ & 25 & 24 & 25 & 25 & 25 & 22 & 21 & 24 & 24 & 25 & 25 & 25 & \\
\hline A & 9 & 11 & 7 & 7 & 8 & 5 & 6 & 12 & 8 & 7 & 5 & 7 & 92 \\
\hline ho & 1.00 & 0.88 & 0.12 & 0.76 & 0.28 & 0.73 & 0.48 & 0.75 & 0.46 & 0.72 & 1.00 & 0.80 & \\
\hline he & 0.84 & 0.88 & 0.74 & 0.82 & 0.87 & 0.75 & 0.58 & 0.87 & 0.80 & 0.68 & 0.76 & 0.73 & \\
\hline \multicolumn{14}{|l|}{ LP } \\
\hline $\mathrm{n}$ & 30 & 30 & 28 & 30 & 30 & 28 & 30 & 28 & 29 & 30 & 29 & 30 & \\
\hline A & 11 & 10 & 5 & 7 & 10 & 9 & 7 & 11 & 6 & 8 & 9 & 7 & 100 \\
\hline ho & 0.63 & 0.77 & 0.00 & 0.47 & 0.37 & 0.46 & 0.47 & 0.86 & 0.62 & 0.73 & 0.59 & 0.40 & \\
\hline he & 0.89 & 0.86 & 0.71 & 0.73 & 0.86 & 0.69 & 0.74 & 0.88 & 0.68 & 0.78 & 0.83 & 0.72 & \\
\hline \multicolumn{14}{|l|}{ LLJ } \\
\hline $\mathrm{n}$ & 22 & 21 & 20 & 22 & 20 & 20 & 20 & 20 & 19 & 22 & 22 & 21 & \\
\hline A & 14 & 11 & 2 & 6 & 10 & 11 & 6 & 10 & 6 & 8 & 8 & 7 & 99 \\
\hline ho & 0.82 & 0.71 & 0.00 & 0.68 & 0.50 & 0.85 & 0.45 & 0.80 & 0.68 & 0.73 & 0.59 & 0.43 & \\
\hline he & 0.89 & 0.87 & 0.47 & 0.71 & 0.79 & 0.90 & 0.64 & 0.84 & 0.65 & 0.84 & 0.73 & 0.77 & \\
\hline $\mathrm{n}_{\text {Total }}$ & 77 & 75 & 73 & 77 & 75 & 70 & 71 & 72 & 72 & 77 & 76 & 76 & \\
\hline $\mathrm{A}_{\text {Total }}$ & 15 & 16 & 9 & 10 & 14 & 13 & 9 & 13 & 11 & 11 & 9 & 10 & 140 \\
\hline
\end{tabular}

$\mathrm{n}$ = número de individuos con genotipo conocido por población; $\mathrm{n}_{\text {Total }}=$ número total de individuos con genotipo conocido; $\mathrm{A}=$ número de alelos detectados en cada población; $\mathrm{A}_{\text {Total }}=$ número de alelos detectados en total; ho = heterocigosidad observada; he $=$ heterocigosidad esperada

$\mathrm{n}=$ number of individuals genotyped per population; $\mathrm{n}_{\text {Total }}=$ total number of individuals genotyped; $\mathrm{A}=$ number of alleles detected in each population; $\mathrm{A}_{\text {Total }}=$ total number of alleles detected; ho= observed heterocigosity; he $=$ expected heterocigosity 
mencionar que el $40 \%$ de dichas variantes presentaron frecuencias génicas $>5 \%$. Por otra parte, en las poblaciones de LIJ y LP se hallaron los alelos 183 (LAB1), 135 (LAB3), 176 (LAB6), 167, 169 (LCA65) con frecuencias que varían desde 0,13 a 0,35 , mientras que en la población de SI estos alelos no se detectaron. Los alelos 169, 173 y 175 (LAB1), 125 (LAB3), 160 (LAB14), 159, 161, 163 (LCA65) están presentes en las tres poblaciones aunque con frecuencias considerablemente mayores en la población de SI. Diecinueve de las treinta y seis pruebas de $\mathrm{H}-\mathrm{W}$, medidas según la prueba exacta (Guo \& Thompson 1992), mostraron desvíos significativos $(\mathrm{P}<0,05)$. En todos los casos estas desviaciones se debieron a un déficit de heterocigotos (Tabla 3). Los loci LAB6 y LAB 13 presentaron probabilidades de existencia de alelos nulos de 0,893 y 0,402 respectivamente en las tres poblaciones por lo que se recalculó el equilibrio de HardyWeinberg excluyendo del análisis a dichos loci (datos no mostrados). Estos cálculos no modificaron los valores de EHW por lo que se descarta la existencia de alelos nulos como causa del desequilibrio por exceso de homocigotos.

TABLA 2

Diversidad genética en términos de heterocigosidad observada (Ho) y esperada (He) media, variabilidad alélica media por locus (NMA) y alelos privados por población (APP)

Genetic diversity in terms of mean observed heterocigosity (Ho) and expected heterocigosity ( $\mathrm{He})$, mean allelic variability by locus (MNA) and private alleles by population (APP)

\begin{tabular}{lcccc}
\hline Población & NMA & APP & $\mathrm{H}_{\mathrm{e}}$ & $\mathrm{H}_{\mathrm{o}}$ \\
\hline SI & 7.67 & 15 & 0.78 & 0.66 \\
LP & 8.33 & 11 & 0.78 & 0.53 \\
LLJ & 8.25 & 20 & 0.76 & 0.60 \\
\hline
\end{tabular}

El estadístico $\mathrm{F}_{\mathrm{ST}}$ total $(=0,07 ; \mathrm{P}=0.00)$ calculado para todos los loci y para todos los planteles de llamas indica un grado moderado de subdivisión poblacional. Según este parámetro el $93 \%$ de la varianza en las frecuencias alélicas se expresa dentro de cada población y solo el $7 \%$ de la varianza se atribuye a diferencias entre las poblaciones. El valor $F_{I S}$ Total $(0,24)$ indica una importante deficiencia de heterocigotos dentro de cada plantel; este índice es una medida indirecta de consanguinidad y concuerda con los resultados obtenidos mediante las pruebas exactas de EHW (Tabla 3). Estos valores indican un alejamiento de las condiciones esperadas en una población cuyos cruzamientos se realizan al azar. Al considerar el pool génico de las tres poblaciones (SI, LP, LLJ), se observa un déficit mayor de genotipos heterocigotos $\left(\mathrm{F}_{\mathrm{IT}}=0,29\right)$, lo que podría señalar cierta estructuración poblacional (Tabla 4).

Cuando se analizaron los planteles mediante el estadístico Rho se observaron algunas diferencias. El $\mathrm{R}_{\mathrm{ST}}$ total $(0,12)$ indica un grado de diferenciación poblacional moderadamente alto, del cual se infiere que el $88 \%$ de la varianza total se encuentra dentro de cada población y el $12 \%$ restante se atribuye a diferencias entre las poblaciones (Tabla 5). En las Tablas 4 y 5 se observan algunos loci que aportan mayoritariamente a tales niveles de diferenciación. Los resultados obtenidos mediante los dos métodos de cálculo del Análisis Molecular de la Varianza (AMOVA) fueron similares a los valores observados mediante los estadísticos $\mathrm{F}_{\mathrm{ST}}$ y $\mathrm{Rho}_{\mathrm{ST}}(\mathrm{FST}=$ $0,076 ; \mathrm{P}=0,000 ;$ RhoST $=0,158 ; \mathrm{P}=0,000)$.

\section{DISCUSIÓN}

Desde el punto de vista de la diversidad alélica las tres poblaciones analizadas muestran, en general, una variabilidad genética alta. Los resultados de $\mathrm{H}_{\mathrm{o}}$ son similares a los observados en otras especies de camélidos analizadas mediante el uso de marcadores microsatélites (Penedo et al. 1998, 1999a, 1999b, Sasse et al. 2000, Sarno et al. 2001, Wheeler et al. 2001). Además, como en otros estudios poblacionales de vicuñas y guanacos (Wheeler et al. 2001, Sarno et al. 2004, Maté et al. 2005), se observaron desvíos significativos del equilibrio de $\mathrm{H}-\mathrm{W}$, debido a un exceso de homocigotos. Entre las causales de estos desvíos podrían mencionarse a la práctica de apareamientos dirigidos por el criador (selección artificial), a una subdivisión poblacional o bien a la existencia de alelos nulos (Callen et al. 1991). 
TABLA 3

Equilibrio de Hardy-Weinberg (EHW) evaluado mediante la Prueba exacta calculada para cada locus en cada población bajo la Hipótesis alternativa (H1): "Existe déficit de heterocigotos"

Hardy-Weinberg equilibrium (HWE) evaluated by Exact test for each locus and each population according to H1: "Heterozygotes deficit"

\begin{tabular}{|c|c|c|c|c|c|c|}
\hline & \multicolumn{2}{|c|}{ Santa Isabel } & \multicolumn{2}{|c|}{ Los Pioneros } & \multicolumn{2}{|c|}{ Llampa Jaram } \\
\hline & Valor de $\mathrm{P}$ & $\mathrm{DE}$ & Valor de $\mathrm{P}$ & $\mathrm{DE}$ & Valor de P & DE \\
\hline LAB 1 & 1 & 0 & 0,001 & 0 & 0,024 & 0,007 \\
\hline LAB 3 & 0,520 & 0,018 & 0,159 & 0,011 & 0,012 & 0,004 \\
\hline LAB 6 & 0 & 0 & 0 & 0 & 0 & 0 \\
\hline LAB 7 & 0,012 & 0,002 & 0 & 0 & 0,040 & 0,004 \\
\hline LAB 13 & 0 & 0 & 0 & 0 & 0 & 0 \\
\hline LAB 14 & 0,420 & 0,008 & 0,001 & 0,001 & 0,262 & 0,015 \\
\hline LAB 15 & 0,066 & 0,005 & 0 & 0 & 0,012 & 0,002 \\
\hline LAB 16 & 0,111 & 0,014 & 0,205 & 0,015 & 0,265 & 0,017 \\
\hline LAB 17 & 0 & 0 & 0,239 & 0,009 & 0,721 & 0,013 \\
\hline LCA 56 & 0,722 & 0,012 & 0,097 & 0,008 & 0,093 & 0,007 \\
\hline LCA 65 & 1 & 0 & 0 & 0 & 0,059 & 0,007 \\
\hline LCA 77 & 0,787 & 0,105 & 0 & 0 & 0,004 & 0,001 \\
\hline
\end{tabular}

TABLA 4

Estadísticos F calculados para cada loci y para todos los loci (Total) en los tres planteles de llamas F statistics calculated for each loci and all loci (Total) in the three plantel of llamas

\begin{tabular}{lccccccccccccc}
\hline Loci & LAB1 & LAB3 & LAB6 & LAB7 & LAB13 & LAB14 & LAB15 & LAB16 & LAB17 & LCA56 & LCA65 & LCA77 & Total \\
\hline $\mathrm{F}_{\text {IS }}$ & 0,08 & 0,10 & 0,94 & 0,18 & 0,56 & 0,15 & 0,31 & 0,07 & 0,18 & 0,06 & 0,07 & 0,27 & 0,24 \\
$\mathrm{~F}_{\text {ST }}$ & 0,04 & 0,05 & 0,12 & 0,07 & 0,04 & 0,07 & 0,03 & 0,05 & 0,06 & 0,05 & 0,15 & 0,12 & 0,07 \\
$\mathrm{~F}_{\text {IT }}$ & 0,12 & 0,14 & 0,95 & 0,24 & 0,58 & 0,20 & 0,33 & 0,11 & 0,23 & 0,10 & 0,21 & 0,36 & 0,29 \\
\hline
\end{tabular}

TABLA 5

Estadísticos Rho calculados para cada loci y para todos los loci (Total) en los tres planteles de llamas

Rho statistics calculated for each loci and all loci (Total) in the three plantel of llamas

\begin{tabular}{|c|c|c|c|c|c|c|c|c|c|c|c|c|c|}
\hline Loci & LAB 1 & LAB3 & LAB6 & LAB7 & LAB13 & LAB 14 & LAB 15 & LAB 16 & LAB 17 & LCA56 & LCA65 & LCA77 & Total \\
\hline $\mathrm{R}_{\mathrm{IS}}$ & 0,25 & 0,09 & 0,93 & 0,22 & 0,40 & 0,11 & 0,11 & 0 & 0 & 0,07 & 0,07 & 0,02 & 0,14 \\
\hline $\mathrm{R}_{\mathrm{ST}}$ & 0,08 & 0 & 0,10 & 0,01 & 0,23 & 0,12 & 0,02 & 0,06 & 0,03 & 0,06 & 0,57 & 0,17 & 0,12 \\
\hline $\mathrm{R}_{\mathrm{IT}}$ & 0,31 & 0,08 & 0,94 & 0,23 & 0,54 & 0,22 & 0,13 & 0 & 0,02 & 0,13 & 0,60 & 0,19 & 0,25 \\
\hline
\end{tabular}


Es probable que el exceso de homocigotos sea el resultado de la selección artificial practicada mediante el manejo reproductivo. La selección en estos animales consiste en la utilización de dos a tres machos reproductores, seleccionados por calidad de fibra y el castrado del resto; si bien se sabe que al momento de establecerse las tropas se introdujeron animales de otras poblaciones, en los 10 años siguientes cada unidad de producción se reprodujo en forma cerrada. Otro aspecto a tenerse en cuenta es el comportamiento reproductivo natural y la organización social de esta especie poligínica. La organización social de las llamas es similar a la de los guanacos silvestres, la que consiste de rebaños formados por machos, hembras y sus crías, donde se establecen jerarquías sociales con un macho dominante que controla el acceso de otros machos a su territorio de reproducción, alimento y bebida. Cada rebaño tiene un territorio permanente establecido por un macho reproductor, quien expulsa a las crías macho antes de que cumplan un año de edad y retiene a las hembras para asegurar el crecimiento del rebaño (Franklin 1992). Este comportamiento de exclusión de machos jóvenes e inclusión de hembras por parte del macho dominante, que es conservado en las poblaciones bajo manejo (Lamas comunicación personal) y resultaría en un déficit de heterocigotos. Por otra parte, no se puede excluir la existencia probable de alelos nulos. Los heterocigotos nulos tienden a contabilizarse como homocigotos, derivando en un déficit de heterocigotos que desvía el EHW. Cuando se analizó el EHW por marcador, algunos de los microsatélites fuera del equilibrio mostraron alta probabilidad de alelo nulo. Los alelos nulos se producen cuando ocurre una mutación en la secuencia complementaria de los primers, impidiendo la amplificación por PCR de uno o de ambos alelos. Para confirmar o descartar esta hipótesis, es necesario diseñar nuevos primers y tipificar nuevamente los loci dudosos (Callen et al. 1993, Koorey et al. 1993, Pemberton et al. 1995). De todas maneras, cuando se recalcularon las pruebas para medir el EHW excluyendo del análisis a los loci LAB6 y LAB13, se observaron los mismos resultados que los obtenidos para el total de los loci.

Como se mencionó anteriormente, varios trabajos de caracterización de camélidos sudamericanos realizados con diferentes microsatélites (Sarno et al. 2001, Wheeler et al. 2001, Maté et al. 2005), detectaron un marcado déficit de heterocigotos. Estos resultados apoyarían la propuesta que los desvíos observados se deberían a factores poblacionales como la estructura social de estas especies y no un artefacto de la metodología utilizada. El conocimiento histórico de las poblaciones de llamas analizadas en este trabajo indica ausencia de flujo genético entre ellas en los últimos 10 años, sea por cuestiones geográficas o de manejo reproductivo. Por tanto, el incremento en la frecuencia de algunos alelos en la población de SI sugeriría la existencia de deriva genética, probablemente debida al tamaño efectivo pequeño del núcleo fundador (Nabata et al. 2004). Cuando la población es pequeña, el número de gametas que contribuyen en la generación siguiente también es pequeño y está sujeto a desviaciones de muestreo dependientes del tamaño efectivo de la población $\left(\mathrm{N}_{\mathrm{e}}\right)$ o del número total de padres que contribuyen a la siguiente generación. La fertilización al azar de las gametas masculinas y femeninas puede modificar la frecuencia genética de una generación a la siguiente. Este fenómeno puede provocar la fijación o pérdida de variantes alélicas en el reservorio genético como resultado del azar (Cavalli-Sforza 1996, Nei 2000).

Los dos índices de subdivisión poblacional empleados mostraron un grado de diferenciación genética moderado, aunque significativo, entre las tres poblaciones. Además, la diferencia observada entre los valores obtenidos con los estadísticos $\mathrm{F}_{\mathrm{ST}} \mathrm{y}$ $\mathrm{R}_{\mathrm{ST}}$, indicarían que estas diferencias serían consecuencia tanto de las variaciones en las frecuencias génicas como en el tamaño de los alelos presentes. Esto podría explicarse por la presencia de alelos privados en cada población, los cuales podrían proceder de la población original o haber ingresado mediante la introducción de reproductores de diferentes regiones geográficas del país y de países vecinos. Comparando con los datos obtenidos en otros mamíferos superiores nuestras observaciones en las llamas son equivalentes a las existentes en otras especies domésticas (Kantanen et al. 2000, Cañón et al. 2001, Mateus et al. 2004). Sin embargo, si se las compara con estudios realizados en guanacos 
silvestres de la Patagonia argentina, los valores de diferenciación genética son menores debido, probablemente, a un mayor flujo genético entre los guanacos (Maté et al. 2005). En otro extremo se encuentran las poblaciones de vicuñas de Chile y Perú donde la diferenciación genética es alta debido al aislamiento geográfico experimentado por las poblaciones de esta especie durante muchísimos años (Wheeler et al. 2001, Sarno et al. 2004).

Los recursos genéticos animales constituyen un patrimonio de inestimable valor. La pérdida de diversidad genética disminuye la capacidad de mantener y/o mejorar la producción pecuaria sostenible, reduciendo la aptitud para hacer frente a nuevos desafíos ambientales (Food and Agricultural Organization of the United Nations - FAO-, http://fao.org/dad-is).

Estudios estadísticos realizados por la FAO en 1995, estiman que un $30 \%$ de las razas de animales domésticos corren riesgo de extinción y cada mes se pierden aproximadamente unas seis razas, con el agravante que el $50 \%$ de las mismas se encuentran en países desarrollados.

A la luz de los resultados aportados por este trabajo, las poblaciones de llamas actuales conservan, en general, una alta variabilidad genética, a pesar de su comportamiento natural, la presión de selección reproductiva y el cuello de botella sufrido por esta especie luego de la colonización de América, situación que probablemente haya llevado al establecimiento de cruzamientos híbridos con la consecuente pérdida de las razas puras (Kadwell et al. 2001).

En un programa de conservación de razas uno de los objetivos perseguidos es el mantenimiento de la mayor cantidad de diversidad genética con un mínimo incremento de la consanguinidad por generación. Desde el punto de vista práctico, para alcanzar el último de los objetivos planteados, se considera prioritario establecer registros genealógicos que permitan optimizar el diseño de apareamientos entre los individuos, principalmente aquellos con alelos privados, para intentar retener la máxima variabilidad genética en las generaciones futuras. Además, el progreso en estudios de variabilidad genética de otras poblaciones de llamas resulta necesario para identificar diferentes reservorios genéticos de los cuales extraer reproductores que puedan mejorar el estado reproductivo de otras poblaciones. Por último, sería de gran utilidad realizar estudios de segregación familiar de marcadores de ADN microsatélite asociados a caracteres de interés económico como calidad de fibra, color de capa, aptitud reproductiva, etc. y aplicarlos a la obtención de mapas genéticos que en el futuro permitan realizar mejoramiento genético más rápido y eficiente asistido por marcadores moleculares.

\section{AGRADECIMIENTOS}

Los autores agradecen a los criadores de llama por la provisión de las muestras de sangre empleadas en esta investigación y a M Silbestro por su asistencia técnica. El presente trabajo se realizó con subsidios de Agencia Nacional de Promoción Científica y Técnica (ANPCyT), Consejo Nacional de Investigaciones Científicas y Técnicas (CONICET) y Comisión de Investigaciones Científicas de la Provincia de Buenos Aires (CICPBA) de Argentina.

\section{LITERATURA CITADA}

ARANGUREN MÉNDEZ J, J JORDANA \& M GÓMEZ (2001) Genetic diversity in spanish donkey breeds using micrisatellite DNA markers. Genetics Selection and. Evolution 33: 243-252.

BUSTAMANTE AV, A ZAMBELLI \& L VIDAL-RIOJA (1999) Analysis of genetic variation in llama (Lama glama) by RAPD markers. Journal of Camel Practice and Research 6: 271-273.

BUSTAMANTE AV, A ZAMBELLI, D DE LAMO, J VON THUNGEN \& L VIDAL-RIOJA (2002) Genetic variability of guanaco and llama populations in Argentina. Small Ruminant Research 44: 97-101.

BUSTAMANTE AV, ML MATÉ, A ZAMBELLI \& L VIDAL-RIOJA (2003) Isolation and characterization of 10 polymorphic dinucleotide microsatellite markers for llama and guanaco. Molecular Ecology Notes 3: 68-69.

CALLEN DF, AD THOMPSON, Y SHEN, HA PHILLIPS, RI RICHARDS, JC MULLEY \& GR SUTHERLAND (1993) Incidence and origin of "null" alleles in the (AC)n microsatellite markers. American Journal of Human Genetics 52: 922-927.

CAÑÓN J, ALEXANDRINO P, BESSA I, CARLEOS C, CARRETERO Y, DUNNER S, FERRAN N, GARCÍA D, JORDANA L, LALOE D, PEREIRA A, SÁNCHEZ A \& MOAZAMI-GOUDARZI K (2001) Genetic diversity measures of local European beef cattle breeds for conservation purpose. Genetics Selection and Evolution 33: 311389.

CAVALLI-SFORZA L (1966) Population structure and human evolution. Proceedings of the Royal Society of London 164: 362-379. 
EXCOFFIER L, PE SMOUSE \& JM QUATTRO (1992) Analysis of molecular variance inferred from metric distance among DNA haplotypes: application of human mitochondrial DNA restriction date. Genetics 136: 343-359.

FAO (1995) World watch list for domestic animal diversity. Second edition. Food and Agricultural Organization of the United Nations, FAO, Rome, Italy. http://fao.org/dad-is.

FRANKLIN W (1992) Biology, ecology and relationship to man of the South American Camelids. In: Mares MA \& H Genoways (eds) Mammalian biology of South America: 457-489. The Pymatuning Symposia in Ecology, University of Pittsburgh, Linesville, Pennsylvania, USA.

GONZÁLEZ-CANDELAS \& MONTOLIO A (2000) Genetic differentiation and structure of Hippocrepis valentina (Leguminosae) populations. Journal of Heredity 91: 134-141.

GUO SW \& EA THOMPSON (1992) Performing the exact test of Hardy-Weinberg proportions for multiple alleles. Biometrics 48: 361-372.

KADWELL M, M FERNÁNDEZ, HF STANLEY, R BALDI, JC WHEELER, R ROSADIO \& MW BRUFORD (2001) Genetic analysis reveals the wild ancestors of the llama and the alpaca. Proceeding of Royal Society of London 268: 2575-2584.

KANTANEN J, OLSAKER I, HOLM LE, LIEN S, VILKKI J, BRUSGAARD K, EYTHORSDOTTIR E, DANELL B \& ADALSTEINSSON S (2000) Genetic diversity and population structure of 20 North European cattle breeds. Journal of Heredity 91: 446-457.

KOOREY DJ, GA BISHOP \& GW MCCAUGHN (1993) Allele non-amplification: a source of confusion in linkage studies employing microsatellite polymorphisms. Human Molecular Genetics 2: 289291.

MATÉ ML, AV BUSTAMANTE, G GIOVAMBATTISTA, D DELAMO, H LAMAS, J VON THUNGEN, A ZAMBELLI \& L VIDAL-RIOJA (2005) Genetic diversity and differentiation of guanaco populations from Argentina inferred from microsatellite data. Animal Genetics 36: 316-321.

MARSHALL T, J SLATE, L KRUUK \& J PEMBERTON (1998) Statistical confidence for likelihood-based paternity inference in natural populations. Molecular Ecology 7: 639-655.

MATEUS JC, PENEDO MC, ALVES VC, RAMOS M \& RANGEL-FIGUEIREDO T (2004) Genetic diversity and differentiation in Portuguese cattle breeds using microsatellites. Animal Genetics 35: 106-113.

NABATA D, R MASUDA \& O TAKAHASHI (2004) Bottleneck effects on the sika deer Cervus nippon population in Hokkaido, revealed by ancient DNA analysis. Zoological Science 21: 473-481.

NEI M \& S KUMAR (2000) Molecular evolution and phylogenetics. Oxford University Press, New York, New York, USA. 333 pp.

NOVOA C \& JC WHEELER (1984) Llama y alpaca. En: Mason IL (ed) Evolution of domesticated animals: 116-128. Longman, London, United Kingdom.

PARK S (2001) MStools v 3 (Excel spreadsheet toolkit for data conversion) Smurfit Institute of Genetics, Trinity College, Dublin 2, Ireland.

PEMBERTON JM, J SLATE, DR BANCROFT \& JA BARRET (1995) Nonamplifying alleles at microsatellite loci: a caution for parentage and population studies. Molecular Ecology 4: 249-252.

PENEDO MCT, AR CAETANO \& KI CORDOVA (1998) Microsatellite markers for South American camelids. Animal Genetics 29: 411-412.

PENEDO MCT, AR CAETANO \& KI CORDOVA (1999a) Eight microsatellite markers for South American camelids. Animal Genetics 30: 166-167.

PENEDO MCT, AR CAETANO \& KI CORDOVA (1999b) Six microsatellite markers for South American camelids. Animal Genetics 30: 399.

RICE JA (1995) Mathematical statistics and Data analysis. Second edition. WH Freeman \& Company, New York, New York, USA. $651 \mathrm{pp}$.

RAYMOND M \& F ROUSSET (1995) GENEPOP (v. 1.2): population genetics software for exact test and ecumenicism. Journal of Heredity 86: 248-249.

RAYMOND M \& F ROUSSET (2000) GENEPOP version $3.2 \mathrm{a}$.

SASSE J, M MARIASEGARAM, R BABU, J KINNE \& U WERNERY (2000) South American camelid microsatellite amplification in Camelus dromedaries. Animal Genetics 31: 75-76.

SARNO RJ, WL FRANKLIN, SJ O’BRIEN \& WE JOHNSON (2001) Patterns of mtDNA and microsatellite variation in an island and mainland population of guanacos in southern Chile. Animal Conservation 4: 93-101

SARNO RJ, VILLALBA L, BONACIC C, GONZALEZ B, ZAPATA B, MAC DONALD DW, O'BRIEN SJ \& JONSON WE (2004) Phylogeography and subspecies assessment of vicuñas in Chile and Bolivia utilizing mtDNA and microsatellite markers: implications for vicuña conservation and management. Conservation Genetics 5: 89-102.

WHEELER JC (1983) La domesticación de la alpaca (Lama pacos) y la llama (Lama glama) y el desarrollo temprano de la ganadería autóctona en los Andes centrales. Informe final: 101-111 Lima, Perú.

WHEELER JC (1995) Evolution and present situation of the South American camelids. Biological Journal of the Linnean Society 54: 271-291.

WHEELER JC, M FERNÁNDEZ, R ROSADIO, D HOCES, M KADWELL \& MW BRUFORD (2001) Diversidad genética y manejo de poblaciones de vicuñas en el Perú. Revista Virtual Visión Veterinaria (Perú) 1: 170-183. 